\title{
Comparative Epidemiology and Outcomes among Trauma Patients transported by Private and Formal Pre-hospital Care Modes in Kigali, Rwanda
}

Jean Paul NZABANDORA ( $\nabla$ jpnzaba7@gmail.com )

University of Rwanda College of Medicine and Health Sciences

Saadiyah Bilal

Icahn School of Medicine at Mount Sinai

Ling Jing

Case Western Reserve University School of Medicine

Michael B. Henry

University of Arizona health Education Alliance

Aly Beeman

Warren Alpert Medical School of Brown University: Brown University Warren Alpert Medical School

Gabin Mbanjumucyo

University of Rwanda College of Medicine and Health Sciences

Noah Rosenberg

Warren Alpert Medical School of Brown University: Brown University Warren Alpert Medical School

Jeanne D’Arc Nyinawankusi

Service d'Aide Medicale Urgente

Vinay Sharma

Michigan State University College of Human Medicine

Vincent Ndebwanimana

University of Rwanda College of Medicine and Health Sciences

Jean Muragizi

University of Rwanda College of Medicine and Health Sciences

Adam R. Aluisio

Warren Alpert Medical School of Brown University: Brown University Warren Alpert Medical School

Original Research

Keywords: Trauma, Rwanda, Global health, pre-hospital care, emergency medical service

Posted Date: April 21st, 2021

DOl: https://doi.org/10.21203/rs.3.rs-428668/v1 
License: (c) (i) This work is licensed under a Creative Commons Attribution 4.0 International License. Read Full License 


\section{Abstract}

Introduction

Injuries account for approximately five million deaths worldwide and have high burdens in low- and middle-income countries, where pre-hospital systems are being developed to improve acute injury care. This study compares the characteristics and outcomes between trauma patients transported by formal emergency medical services (EMS), known as Service d'Aide Medicale Urgente, and by private transport to the emergency department (ED) at the Centre Hospitalier Universitaire de Kigali (CHUK) in Rwanda.

\section{Methods}

This retrospective cohort study analysed a random sample of trauma patients from of the CHUK ED presenting between 1 August 2015 and 30 July 2016. Non-trauma patients and transferred patients were excluded. The primary outcomes were ED and inpatient dispositions and outcome characteristics of: admission, length of stay (LOS), mortality. Descriptive analyses were performed and differences in outcomes assessed based on transport modalities via Pearson Chi-Squared or Fisher's Exact tests.

Results

There were 556 trauma patients analysed, of which $87.1 \%$ were transported by private transport and the remainder by EMS. The median age was 28 years (IQR:18-39), 52.1\% had serious injuries, based on the triage system at CHUK. Common mechanisms of injury included road traffic crashes (62.7\%) and falls (17.1\%). Median inpatient LOS for private transport patients was 7 days (IQR:3-16 days), compared to 9.5 days for EMS patients (IQR:4-18 days) $(p=0.301)$. There was a significant relationship between ED disposition and transport method $(p=0.005)$, with a higher number of admissions among EMStransported patients. The observed mortality of EMS and privately transported patients were not significant different, however there were a higher percentage of deaths in the ED and inpatient setting among those who were privately transported.

\section{Conclusion}

While mortality and hospital LOS between EMS and private transport patients were not statistically significantly different, this may not reflect a lack of clinical significance. Future research will be needed to continue to assess the EMS utilisation rate, quality and impact on patient-centred outcomes and should include studies that prospectively investigate larger samples of patients to better evaluate the effects of EMS trauma care in Rwanda.

\section{Introduction}

Injury is a major cause of death and disability globally, accounting for approximately 5 million deaths each year [1]. Although trauma systems can improve patient outcomes, implementation is challenging in low- and middle-income countries (LMICs), where over $80 \%$ of injury-related deaths occur [2]. 
The aim of pre-hospital emergency medical services (EMS) is to provide temporising care and rapid transport to a medical facility. Many LMICs continue to rely on informal means of pre-hospital transport, such as private vehicles and ad-hoc bicycle ambulances, due to the unavailability or inadequacy of formal EMS or frequent delays in transport [2-5]. The data on the benefits of formal pre-hospital transport are mixed [6]; in some studies, EMS with pre-hospital interventions appears to have reduced injury-related mortality [7] while delays in emergency transport services have been linked to increased perinatal deaths in other settings[8]. In some cases, however, the strategy of 'scoop and run', which prioritises expedient transport by lay folk and Basic Life Support crews to definitive facilities-based care, may be superior to 'stay and play', in which personnel with more advanced medical training provide treatment and interventions in the field [6]. While non-EMS transport may result in faster hospital arrival times [9], it is not yet clear in many contexts how this impacts outcomes.

In Rwanda, injuries account for nearly one-quarter of deaths, nearly half of which occur in the pre-hospital setting [10]. In 2007, the Rwandan government created the Service d'Aide Médicale Urgente, (SAMU) an EMS system to provide formal pre-hospital care. The majority of patients served by EMS in Rwanda are victims of traumatic injuries, most frequently road traffic crashes (RTCs), with a $5.5 \%$ mortality rate overall [11]. However, the utilisation of EMS and impacts of the SAMU program on hospital outcomes are not well-defined. The aim of this study was to compare characteristics and outcomes between trauma patients presenting via EMS and those presenting via informal private transport.

\section{Methods}

This retrospective analysis evaluated ED trauma cases presenting from 1 August 2015 to 30 July

2016 at Centre Hospitalier Universitaire de Kigali (CHUK). Inclusion criteria were involvement in trauma and transportation directly from the trauma scene to CHUK. Exclusion criteria were transfers from other hospitals, non-trauma patients, and those lacking documentation for the ED encounter. All eligible patients were stratified into two groups based on whether they were transported by EMS or private transport. The research activities were approved by the CHUK ethics committee (EC/CHUK/513/2018) and the University of Rwanda College of Medicine and Health Sciences (No 421/CMHS IRB/2017).

\section{Study Setting:}

The study site is a tertiary level care centre and public teaching hospital in Kigali, Rwanda that is a main receiving centre for trauma patients in Rwanda and serving a population size of more than 12 million [12]. CHUK provides multiple specialty services, including orthopaedic and neurosurgical interventions, advanced imaging, and laboratory testing; its 24-hour ED ensures extensive acute care for adult and paediatric acutely injured patients.

The EMS system coordinates the prehospital clinical care and provides field stabilization throughout Kigali and various provinces/districts hospitals of Rwanda [13]. As of 2016, there were 225 total ambulances in operation throughout Rwanda that trained nurses and anesthetists' staff. Patients can 
utilize the EMS system by calling a toll-free number of "912". The national dispatch EM system located at CHUK receives approximately 1200 calls monthly [14].

\section{Data Management:}

This analysis included patients from a previously accrued electronic dataset [15-17]. This dataset was created by using a validated data acquisition approach on the hospital database to identify all cases that presented at the CHUK ED during the accruement period. and then randomly sampling a number of from each month (range: 135-165 records per month) [15]. Personnel utilized a standardized data collection instrument to abstract data for entry into a secure password-protected electronic data management application, ensuring that data procedures conformed to standard quality practices for chart review research in Emergency Medicine [18]. Due to the time-consuming process of data extraction from paper charts to construct the electronic database and its feasibility to review all cases during the time period of interest, a random subset of all encounters was used to represent data of the overall population [15]. As this is a retrospective exploratory analysis aimed at understanding baseline characteristics of the population of interest to inform future research no formal sample size was calculated.

Demographics were collected for each patient, including age, sex, mechanism of injury, and triage severity. Triage severity at CHUK is determined using the South African Triage Scale (SATS) [19, 20], consisting of Green (routine), Yellow (urgent), Orange (very urgent), and Red (emergency) categorizations. At CHUK, the Red triage category is further divided into "alarm", the most severe category, characterised by emergency signs in airway, breathing, circulation, convulsions, or coma, requiring immediate intervention; and "no alarm", determined purely by SATS-specified vital signs. Glasgow Coma Scale (GCS) scores were also collected. Other data gathered included patient transfer method, length of stay (LOS) in the ED and inpatient settings, and in-hospital mortality and outcomes.

\section{Data analysis:}

Descriptive and inferential analyses were completed for the overall cohort and stratified based on whether they were transferred by EMS or private transport. Continuous data was reported as means with standard deviations or medians with interquartile ranges (IQR) and categorical data as frequencies and percentages. Patient characteristics and outcomes were compared between the two transportation method cohorts. The comparative outcomes of interest were need for inpatient admission, length of stay (LOS) and mortality ED. Mann-Whitney tests for continuous measures and Pearson Chi-Squared or Fisher's Exact tests for categorical data were used to assess for significant differences or relationships between groups using a standard p-value cut-off of $<0.05$.

\section{Results}

The database query generated 22,117 unique encounters between August 2015 and July 2016, of which 4,620 cases were randomly selected and screened. Of those, a random sample of 556 trauma patients met inclusion criteria and were analyzed. (Fig. 1). The median age of patients was 28 years (IQR:18-39), and $52.1 \%$ had serious injuries (triaged as Orange or Red based on SATS). Common mechanisms of 
injury included RTCs (62.7\%) and falls (17.1\%). Private transport was used by $87.1 \%$ (484) of the patients, while $12.9 \%$ (72) were transported by EMS.

Among both private and EMS transported patients of all triage severities, RTCs were found to be the leading cause of trauma. There was no significant association between transport method and age, sex, or SATS triage severity $(p=0.190,0.147$, and 0.198 , respectively), although $58.8 \%$ of EMS patients were triaged as severely injured compared to $51.0 \%$ of private transport patients, based on SATS categories of Red or Orange. Mechanisms of injury differed between the groups: EMS-transported patients were more likely to have been involved in RTCs, while private transport patients were more frequently involved in falls or penetrating trauma unrelated to RTCs $(p=0.010)$. The most consulted team for further management was orthopaedics $(45.0 \%)$, followed by acute care surgery $(30.5 \%)$ (Table 1$)$.

Table 1: Demographics of patients brought to ED via formal pre-hospital transport and private transport 


\begin{tabular}{|c|c|c|c|c|}
\hline & & $\begin{array}{l}\text { Private } \\
\text { Transport }\end{array}$ & EMS & $\begin{array}{l}\mathrm{p}- \\
\text { value }\end{array}$ \\
\hline Age (years) & & $29.8 \pm 18.4$ & $\begin{array}{l}32.4 \pm \\
16.2\end{array}$ & 0.190 \\
\hline \multirow[t]{2}{*}{ Sex } & Male & $336(69.4 \%)$ & $\begin{array}{l}56 \\
(77.8 \%)\end{array}$ & \multirow[t]{2}{*}{0.147} \\
\hline & Female & $148(30.6 \%)$ & $\begin{array}{l}16 \\
(22.2 \%)\end{array}$ & \\
\hline \multirow{6}{*}{$\begin{array}{l}\text { South Africa Triage Scale } \\
\text { Categories }\end{array}$} & Red alarm & $8(1.8 \%)$ & $2(2.9 \%)$ & \multirow[t]{6}{*}{0.198} \\
\hline & Red no alarm & $35(7.9 \%)$ & $9(13.2 \%)$ & \\
\hline & Orange & $183(41.3 \%)$ & $\begin{array}{l}29 \\
(42.7 \%)\end{array}$ & \\
\hline & Yellow & $149(33.6 \%)$ & $\begin{array}{l}16 \\
(23.5 \%)\end{array}$ & \\
\hline & Green & $28(6.3 \%)$ & $2(2.9 \%)$ & \\
\hline & Unknown & $40(9.0 \%)$ & $\begin{array}{l}10 \\
(14.7 \%)\end{array}$ & \\
\hline \multirow[t]{8}{*}{ Mechanism } & $\begin{array}{l}\text { Road traffic } \\
\text { crashes }\end{array}$ & $291(60.1 \%)$ & $\begin{array}{l}57 \\
(80.3 \%)\end{array}$ & \multirow[t]{8}{*}{0.010} \\
\hline & Burn & $25(5.2 \%)$ & $0(0 \%)$ & \\
\hline & Fall & $88(18.2 \%)$ & $7(9.9 \%)$ & \\
\hline & Animal injury & $2(0.4 \%)$ & $1(1.4 \%)$ & \\
\hline & Blast & $2(0.4 \%)$ & $1(1.4 \%)$ & \\
\hline & Other blunt & $15(3.1 \%)$ & $0(0 \%)$ & \\
\hline & Other penetrating & $46(9.5 \%)$ & $2(2.8 \%)$ & \\
\hline & Unknown & $15(3.1 \%)$ & $4(5.6 \%)$ & \\
\hline \multirow[t]{3}{*}{ Glasgow Coma Scale } & $3-8$ & $17(4.8 \%)$ & $3(4.5 \%)$ & \multirow[t]{3}{*}{0.987} \\
\hline & $9-12$ & $39(10.9 \%)$ & $7(10.5 \%)$ & \\
\hline & $12-15$ & $301(84.3 \%)$ & $\begin{array}{l}57 \\
(85.1 \%)\end{array}$ & \\
\hline
\end{tabular}

The median ED LOS, 1 day (IQR:0-2) for private transport patients and 1 day (IQR:1-2) for EMS patients, did not differ significantly between the two groups $(p=0.103)$. For admitted patients, median inpatient LOS was 7 days for private transport, (IQR:3-16) and 9.5 days for EMS (IQR: 4-18) also did not differ $(p=0.301)$ significantly. 
There was a statistically significant relationship between ED disposition and patient transport method $(p=0.005)$, with $73.6 \%$ of EMS patients requiring admission compared to $59.5 \%$ of privately transport patients. In the ED, $0.8 \%$ of private transport patients and $1.4 \%$ of EMS-transported patients died. In the inpatient setting, none of the EMS patients died while $5.9 \%$ of the admitted private transport patients died during the course of hospitalization. The relationship between inpatient outcome and transport method was not found to be statistically significant $(p=0.248)$ (Table 2). Overall, the total combined ED and inpatient mortality was $4.5 \%$ among private transport patients and $1.4 \%$ among EMS patients, a difference that was not statistically significant $(p=0.339)$.

Table 2: ED and inpatient dispositions for patients brought via formal and informal pre-hospital transport

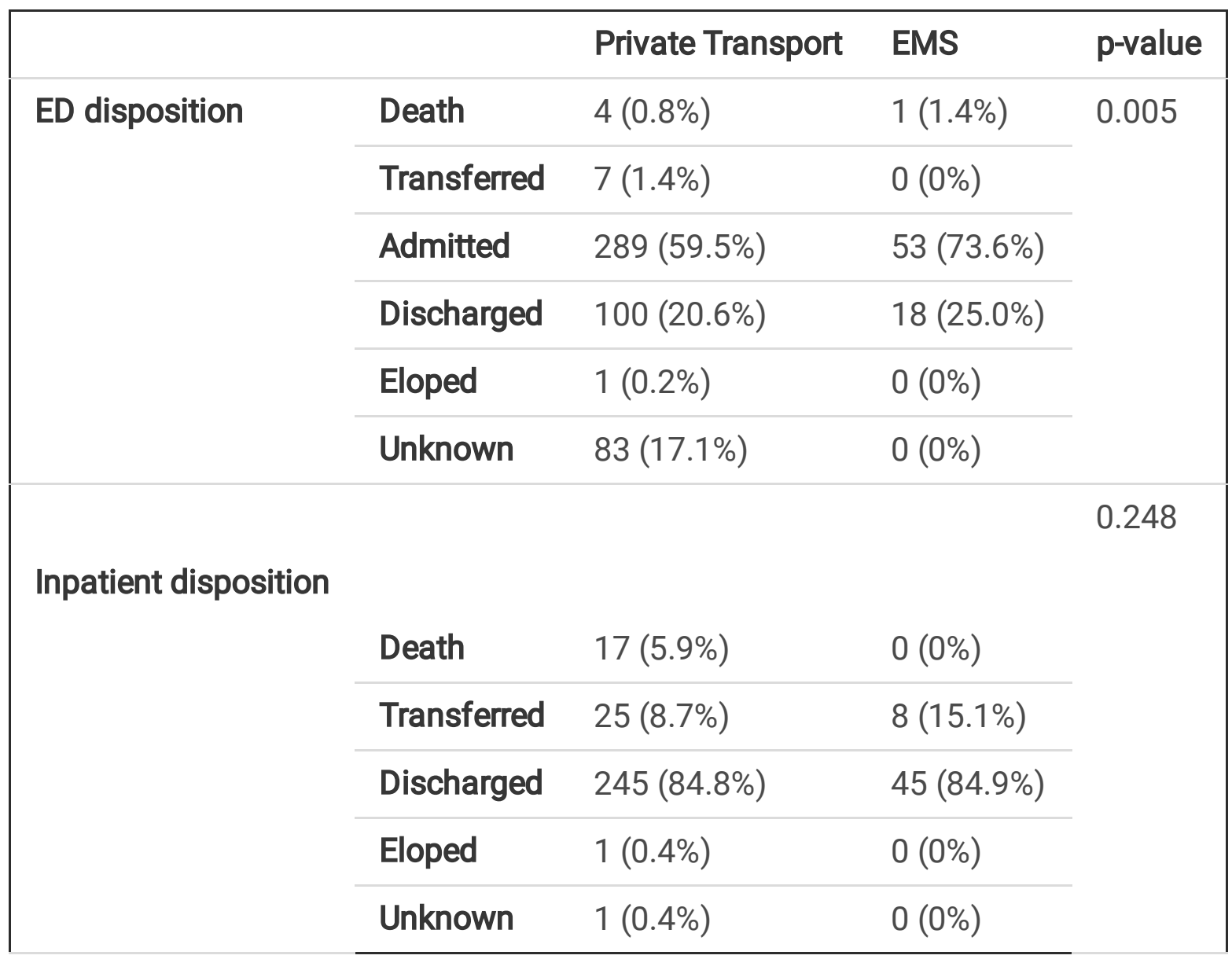

\section{Discussion}

To the knowledge of the authors, this study is among the first from the sub-Saharan African region, specifically Rwanda, comparing associations between patients transported for emergency trauma care by formal EMS and private transport with inpatient and ED dispositions and a focus on hospital resource centred outcomes of length of stay. Similar to prior studies, a higher proportion of trauma patients were brought to the CHUK ED by private transport indicative of a still developing EMS system in Rwanda [21, 22]. Factors such as lack of public knowledge or trust may prevent the Rwandan public from using this government-funded medical resource as it is unclear how widely disseminated the contact information for EMS services is in Rwanda. This is in contrast to neighbouring countries where national and local 
government agencies have made concrete and proactive policy changes in order to widely publicize the contact information for national ambulance services and prioritized calls directed to such services [22].

While the most common injury mechanism was RTCs among both EMS and private transport patients, a significantly greater number of EMS patients were involved in RTCs which has been reported in prior studies as well $[23,24]$. The higher proportion of EMS calls for RTCs may be prompted by the bystanders' perception of the acuity and severity of such traumatic injuries, in contrast to falls, which may occur in more enclosed spaces such as homes and not be witnessed by a gathered public and penetrating trauma, which were more common in private transport patients. The proportion of injuries due to RTCs is higher in this study than has been found in other sub-Saharan countries [25], perhaps reflecting ongoing rapid modernisation and increased vehicle usage in Rwanda.

There was a relationship between the transport method and ED disposition, suggesting that the type of pre-hospital transport could have an effect on immediate patient outcomes. A higher rate of hospital admission among EMS patients suggests that EMS may be responding to more complex and severe injuries and therefore being used appropriately. Although inpatient mortality was higher among private transport patients than EMS patients, this difference was not statistically significant, and overall, there was no relationship between inpatient disposition and transport method. The inpatient LOS appeared to be longer among EMS patients, but with wide variation, this difference was also not statistically significant. Other studies have also shown that while trauma system development may improve outcomes on the national level [7], individual patients with formal EMS transport do not always have improved morbidity, mortality, and LOS compared to those transported by private transport with EMS versus non-EMS transport not showing any difference related to in-hospital mortality $[6,9,23]$. However, the pattern of higher admission rates, lower mortality rates, and longer LOS among EMS patients in our study suggest clinical significance. Similar findings have been reported by studies in Ghana and South Africa with ambulances arriving at a trauma referral centre were transporting a larger percent of higher acuity and critically injured patients than private transport methods [22, 23]. Practically, these differences could indicate that given the vital role of EMS within healthcare systems, patients being transported by this method stand to benefit from prehospital stabilization by trained healthcare personnel to improve and optimize clinical outcomes, although this would need to be verified through future studies with a larger patient population. Future research would be beneficial to characterise EMS utilisation among the Rwandan public and identify factors in formal and informal pre-hospital transport, such as hospital arrival days and times, that are associated with differences in patient outcomes. Studies could also evaluate the effect of quality improvement measures in EMS, such as standardised checklists and protocols, on patient outcomes. As private transport methods will also continue to be used in Rwanda into the foreseeable future, training laypeople in basic emergency care and evaluating patient outcomes, as has been trialled in neighbouring Uganda [24], could be beneficial while EMS continues to develop.

\section{Limitations:}


As this study was retrospective, it was limited by the quality and accuracy of available data. While group characteristics appeared roughly similar, this study could not account for all possible confounding factors, including prior medical history or chronic conditions, involvement of substance use in the trauma mechanism, quality of care received at the scene, and length of time between the accident and arrival at the hospital. However, as this study was primarily explorative, these factors can and should be examined in future studies. Due to the small sample size of EMS patients, significant differences between EMS and private transport patients may not have been identified. Additionally, a difference was found between EMS and private transport patients in terms of ED disposition, but this study did not identify in which dispositions the differences lay. Finally, the time frame of data accruement, while the most recent data available at the time of collection, is limited by a previously built database and may not reflect the current situation in Rwanda's rapidly developing healthcare system. In the future, as Rwanda may contemplate the switch to more electronic-based charting, this study could be more easily updated.

\section{Conclusion}

In conclusion, this study demonstrates that the majority of trauma patients seeking ED care at CHUK are not yet transported by the formal EMS pre-hospital system, but those transported by EMS are admitted more frequently. While there is a relationship between transport method and ED outcomes, transport method in the current data was not significantly associated with mortality or hospital LOS, although this may not reflect clinical significance. As EMS grows and more patients use the service in Rwanda, further research will need to assess its utilisation rate, quality, and impact on patient-centred outcomes.

\section{Abbreviations}

Centre Hospitalier Universitaire de Kigali (CHUK)

emergency department (ED)

emergency medical services (EMS)

interquartile ranges (IQR)

length of stay (LOS)

low- and middle-income countries (LMICs)

road traffic crashes (RTCs)

Service d'Aide Medicale Urgente (SAMU)

\section{Declarations}


Ethics approval: The study was approved by the University Teaching Hospital of Kigali Ethics Committee (EC/CHUK/513/2018) and the University of Rwanda (No 421/CMHS IRB/2017). No personally identifiable information was collected.

Consent to Participate: Not applicable.

Consent for Publication: Not applicable.

Availability of data and materials: The dataset generated and used during the current study is available from the corresponding author on reasonable request.

Competing interests: The authors declare that they have no competing interests. The content of this manuscript is solely the responsibility of the authors and does not necessarily represent the views of or any academic organizations.

Funding: This work was not supported by any funding agency.

Authors' Contributions: JPN, MBH and LJ conceptualized the study, in collaboration with NR, ARA, GB and JDN, VN, JDN. LJ, MBH, and $A B$ analyzed and interpreted the data with supervision from ARA. JPN, LJ and $A B$ drafted the manuscript. VSK, MBH, GN, JM, and ARA revised the manuscript for its scientific content. $A B$ and JPN prepared the manuscript for submission. All authors read and approved the final manuscript.

Acknowledgements: Not applicable

\section{References}

[1] Lozano R, Naghavi M, Foreman K, et al. Global and regional mortality from 235 causes of death for 20 age groups in 1990 and 2010: a systematic analysis for the Global Burden of Disease Study 2010. Lancet 2012;380(9859):2095-2128. https://doi.org/10.1016/S0140-6736(12)61728-0.

[2] Kobusingye OC, Hyder AA, Bishai D, et al. Emergency medical systems in low- and middle-income countries: recommendations for action. Bull World Health Organ 2005;83(8):626-631.

[3] Jayaraman S, Mabweijano JR, Lipnick MS, et al. Current patterns of prehospital trauma care in Kampala, Uganda and the feasibility of a lay-first-responder training program. World J Surg 2009;33(12):2512-21. doi: 10.1007/s00268-009-0180-6.

[4] Cannoodt L, Mock C, Bucagu M. Identifying barriers to emergency care services. Int J Health Plann Manage 2012;27(2):e104-20. doi: 10.1002/hpm.1098.

[5] Siddiqui M, Siddiqui SR, Zafar A, et al. Factors delaying hospital arrival of patients with acute stroke. J Pak Med Assoc 2008;58(4):178-82. 
[6] Ryynänen OP, lirola T, Reitala J, et al. Is advanced life support better than basic life support in prehospital care? A systematic review. Scand J Trauma Resusc Emerg Med 2010;18(62):1-14. doi: 10.1186/1757-7241-18-62.

[7] Henry JA, Reingold AL. Prehospital trauma systems reduce mortality in developing countries: A systematic review and meta-analysis. J Trauma Acute Care Surg 2012;73(1):261-268. doi:

10.1097/TA.0b013e31824bde1e.

[8] Jammeh A, Sundby J, Vangen S. Barriers to emergency obstetric care services in perinatal deaths in rural Gambia: a qualitative in-depth interview study. ISRN Obstet Gynecol 2011. doi: 10.5402/2011/981096.

[9] Cornwell EE, Belzberg H, Hennigan K, et al. Emergency Medical Services (EMS) vs Non-EMS Transport of Critically Injured Patients. Arch Surg 2000;135(3):315-219. doi: 10.1001/archsurg.135.3.315.

[10] Kim WC, Byiringiro JC, Ntakiyiruta G, et al. Vital Statistics: Estimating Injury Mortality in Kigali, Rwanda. World J Surg 2016;40(1): 6-13. doi: 10.1007/s00268-015-3258-3.

[11] Mbanjumucyo G, George N, Kearney A, et al. Epidemiology of injuries and outcomes among trauma patients receiving prehospital care at a tertiary teaching hospital in Kigali, Rwanda. Afr J Emerg Med 2016;6(4):191-197. doi: 10.1016/j.afjem.2016.10.001.

[12] Size of the resident population. National Institute of Statistics of Rwanda. Accessed 7 September 2020. https://www.statistics.gov.rw/publication/size-resident-population.

[13] Enumah, S., Scott, J. W., Maine, R., Uwitonze, E., D’Arc Nyinawankusi, J., Riviello, R., Byiringiro, J. C., Kabagema, I., \& Jayaraman, S. (2016). Rwanda's Model Prehospital Emergency Care Service: A Two-year Review of Patient Demographics and Injury Patterns in Kigali. Prehospital and Disaster Medicine, 31(6), 614-620. https://doi.org/10.1017/S1049023X16000807

[14] Scott, J. W., Nyinawankusi, J. D. A., Enumah, S., Maine, R., Uwitonze, E., Hu, Y., Kabagema, I., Byiringiro, J. C., Riviello, R., \& Jayaraman, S. (2017). Improving prehospital trauma care in Rwanda through continuous quality improvement: an interrupted time series analysis. 5-7.

[15] Aluisio AR, Barry MA, Martin KD, et al. Impact of emergency medicine training implementation on mortality outcomes in Kigali, Rwanda: An interrupted time-series study. Afr J Emerg Med 2019;9(1):14-20. doi: 10.1016/j.afjem.2018.10.002.

[16] Kearney AS, Kabeja LM, George N, et al. Development of a trauma and emergency database in Kigali, Rwanda. Afr J Emerg Med 2016;6(4):185-190. doi: 10.1016/j.afjem.2016.10.002.

[17] Aluisio AR, Garbern S, Wiskel T, et al. Mortality outcomes based on ED qSOFA score and HIV status in a developing low income country. Am J Emerg Med 2018;36(11):2010-2019. doi:

10.1016/j.ajem.2018.03.014.

Page 12/14 
[18] Kaji AH, Schriger D, Green S. Looking through the retrospectoscope: reducing bias in emergency medicine chart review studies. Ann Emerg Med 2014;64(3):292-8. doi:

10.1016/j.annemergmed.2014.03.025.

[19] South African Triage Group, A division of the Emergency Medicine Society of South Africa. South African Triage Score (SATS), 2008. <www.triagesa.co.za>

[20] Uwamahoro C, Aluisio AR, Chu E, et al. Evaluation of a modified South African Triage Score as a predictor of patient disposition at a tertiary hospital in Rwanda. Afr J Emerg Med 2019. doi.org/10.1016/j.afjem.2019.10.001

[21] Hanewinckel R, Jongman HP, Wallis LA, et al. Emergency medicine in Paarl, South Africa: a crosssectional descriptive study. Int J Emerg Med 2010;3(3):143-150. doi: 10.1007/s12245-010-0185-9

[22] Mould-Millman CN, Rominski S, and Oteng R. Ambulance or taxi? High acuity prehospital transports in the Ashanti region of Ghana. Afr J Emerg Med 2014;4(1):8-13. doi.org/10.1016/j.afjem.2013.04.004

[23] Möller A, Hunter L, Kurland L, et al. The association between hospital arrival time, transport method, prehospital time intervals, and in-hospital mortality in trauma patients presenting to Khayelitsha Hospital, Cape Town. Afr J Emerg Med 2018;8(3):89-94. doi: 10.1016/j.afjem.2018.01.001.

[24] Jayaraman S, Mabweijano JR, Lipnick MS, et al. Current Patterns of Prehospital Trauma Care in Kampala, Uganda and the Feasibility of a Lay-First-Responder Training Program. World J Surg 2009;33(12): 2512-2521. doi: 10.1007/s00268-009-0180-6.

[25] Vissoci JRN, Shogilev DJ, Krebs E, et al. Road traffic injury in sub-Saharan African countries: A systematic review and summary of observational studies. Traffic Inj Prev 2017;18(7):767-773. doi: 10.1080/15389588.2017.1314470.

\section{Figures}




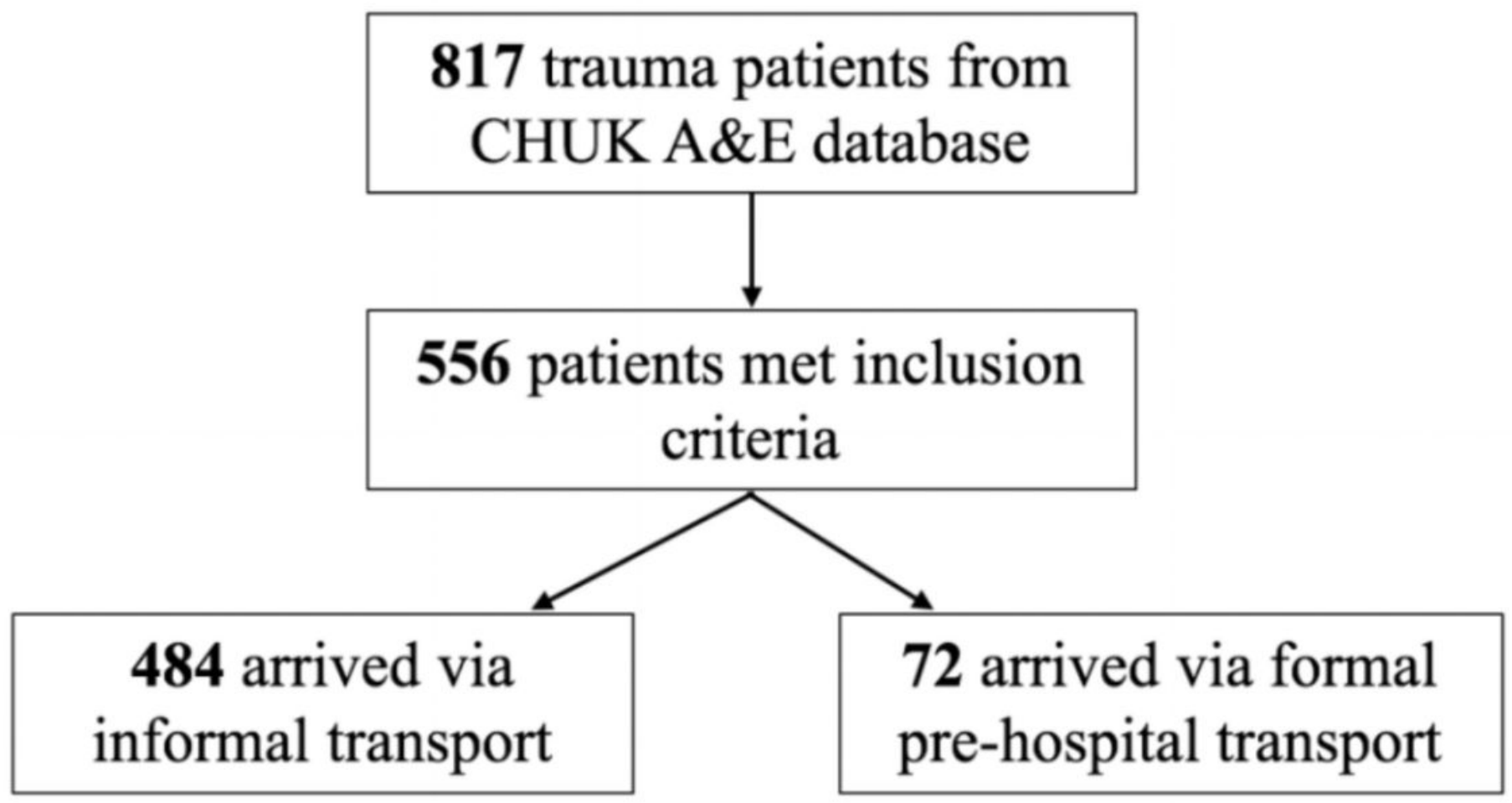

Figure 1

The median age of patients was 28 years (IQR:18-39), and $52.1 \%$ had serious injuries (triaged as Orange or Red based on SATS). Common mechanisms of injury included RTCs (62.7\%) and falls (17.1\%). Private transport was used by $87.1 \%$ (484) of the patients, while $12.9 \%$ (72) were transported by EMS. 